\title{
Silodosin for the treatment of clinical benign prostatic hyperplasia: safety, efficacy, and patient acceptability
}

This article was published in the following Dove Press journal:

Research and Reports in Urology

26 September 2014

Number of times this article has been viewed

\section{Hee Ju Cho \\ Tag Keun Yoo}

Department of Urology, Eulji Hospital, Eulji University School of Medicine, Seoul, Korea
Correspondence: Tag Keun Yoo Department of Urology, Eulji Hospital, Eulji University School of Medicine, 280-I, Hagye I-dong, Nowon-gu, Seoul 139-872, Korea

Tel +82 29708305

Fax +82 29708517

Email ytk5202@eulji.ac.kr
Abstract: $\alpha 1$-Adrenergic receptor antagonists are commonly used to treat male lower urinary tract symptoms and benign prostatic hyperplasia (BPH). We performed a literature search using PubMed, Medline via Ovid, Embase, and the Cochrane Library databases to identify studies on the treatment of BPH by silodosin. Silodosin is a novel $\alpha 1$-adrenergic receptor antagonist whose affinity for the $\alpha 1 \mathrm{~A}$-adrenergic receptor is greater than that for the $\alpha 1 \mathrm{~B}$-adrenergic receptor. Therefore, silodosin does not increase the incidence of blood pressure-related side effects, which may result from the inhibition of the $\alpha 1 \mathrm{~B}$-adrenergic receptor. Patients receiving silodosin at a daily dose of $8 \mathrm{mg}$ showed a significant improvement in the International Prostate Symptom Score and maximum urinary flow rate compared with those receiving a placebo. Silodosin also improved both storage and voiding symptoms, indicating that silodosin is effective, even during early phases of BPH treatment. Follow-up extension studies performed in the United States, Europe, and Asia demonstrated its long-term safety and efficacy. In the European study, silodosin significantly reduced nocturia compared to the placebo. Although retrograde or abnormal ejaculation was the most commonly reported symptom in these studies, only a few patients discontinued treatment. The incidence of adverse cardiovascular events was also very low. Evidence showing solid efficacy and cardiovascular safety profiles of silodosin will provide a good solution for the treatment of lower urinary tract symptoms associated with $\mathrm{BPH}$ in an increasingly aging society.

Keywords: $\alpha 1 \mathrm{~A}$-adrenoceptor antagonist, silodosin, benign prostatic hyperplasia, lower urinary tract symptoms

\section{Introduction and background}

Silodosin, a highly selective $\alpha 1$-adrenergic receptor antagonist for the treatment of lower urinary tract symptoms (LUTS), ${ }^{1}$ was developed in 1995 under its original name, KMD-3213. ${ }^{2}$ Thereafter, several in vitro studies in humans have proved the uroselectivity of silodosin, which affects the contraction of the prostatic smooth muscle, ${ }^{3,4}$ to be greater than that of tamsulosin and naftopidil..$^{5}$ Silodosin was approved in Japan in 2006, more recently it has received approval in the United States, Europe, and Korea. ${ }^{6}$ We performed a literature search using PubMed, Medline via Ovid, Embase, and the Cochrane Library databases to identify research articles, preclinical studies, and systematic and general reviews that discuss the pharmacological features, safety, and efficacy of silodosin.

\section{Pharmacodynamics and pharmacokinetics}

Receptor binding studies show that silodosin has a very strong affinity for the $\alpha 1 \mathrm{~A}-$ adrenergic receptor. For example, the affinity of silodosin for the $\alpha 1 \mathrm{~A}$-adrenergic 
receptor is 162 times higher than that for the $\alpha 1 \mathrm{~B}$-adrenergic receptor, and 55 times higher than that for the $\alpha 1 \mathrm{D}$ adrenergic receptor. ${ }^{7}$ Other studies that address native organ selectivity and $\alpha 1$-adrenoceptor sensitivity reveal that the sensitivity of silodosin for the prostate in Japanese white rabbits was 280 times greater than that for the $\alpha 1 \mathrm{~B}$-adrenergic receptor-rich spleen in Sprague Dawley rats, and approximately 50 times greater than that for the $\alpha 1 \mathrm{D}$-adrenergic receptor-rich thoracic aorta in the same species. The selectivity of silodosin for the urethra and bladder trigone is comparable to the prostate. ${ }^{7,8}$

The uroselectivity of silodosin has also been shown in in vivo studies in Sprague Dawley rats. After the administration of anesthesia and then phenylephrine, which increased the intraurethral pressure, several $\alpha$-blockers, including silodosin, tamsulosin, naftopidil, and prazosin were injected to evaluate their effects on the intraurethral pressure and mean blood pressure. Although silodosin suppressed the intraurethral pressure only, tamsulosin hydrochloride $(\mathrm{HCl})$ can affect the intraurethral pressure and mean arterial pressure at a dose similar to that of silodosin. ${ }^{9}$ The $\mathrm{ID}_{50}$ (defined as the dose at which intraurethral pressure is suppressed by $50 \%)(\mu \mathrm{g} / \mathrm{kg})$, which is defined as the dose that can suppress the increase in intraurethral pressure by $50 \%$, was 0.932 for silodosin, 0.400 for tamsulosin $\mathrm{HCl}, 361$ for naftopidil, and 4.04 for prazosin. The $\mathrm{ED}_{15}$ (defined as the dose at which the mean blood pressure is decreased by $15 \%)(\mu \mathrm{g} / \mathrm{kg})$, which is defined as the dose that can decrease the mean arterial pressure by $15 \%$, was 10.9 for silodosin, 0.895 for tamsulosin $\mathrm{HCl}, 48.1$ for naftopidil, and 0.792 for prazosin. Uroselectivity, which was calculated by $\mathrm{ED}_{15} / \mathrm{ID}_{50}$, was highest in the silodosin group (11.7). The ratios were $2.24,0.133$, and 0.196 for tamsulosin, naftopidil, and prazosin, respectively (Table 1$).{ }^{9}$

Silodosin is metabolized by UDP-glucuronosyltransferase2B7 (UGT2B7), alcohol and aldehyde dehydrogenases, and cytochrome P450 3A4 (CYP3A4) pathways, and is excreted in urine (34\%) and feces (55\%). ${ }^{10}$ Therefore, clinicians should not prescribe silodosin for patients who are also receiving
CYP3A4 inhibitors such as ketoconazole and ritonavir, however, it can be prescribed for those taking moderate CYP3A4 inhibitors, such as diltiazem, or phosphodiesterase-5 inhibitors without significant changes in blood pressure and heart rate. ${ }^{11}$ The half-lives of silodosin and its active metabolite, glucuronide conjugate, are 11 and 18 hours, respectively. In patients with moderate hepatic impairment, the pharmacokinetic profiles of silodosin and its metabolite are not different from those without hepatic impairment. However, there are no silodosin pharmacokinetic studies in individuals with severe hepatic dysfunction. Furthermore, in patients with moderate renal impairment, the optimal dose of silodosin has to be titrated, however, in patients with renal failure, it is not recommended. ${ }^{10}$

\section{Clinical safety and efficacy}

Phase III studies of silodosin have been conducted in Japan, the United States, and Europe. ${ }^{12-14}$ We summarize the results from these studies in Tables 2 and 3. The first Phase III randomized, placebo-controlled, double-blind study was conducted at 88 Japanese centers, and it involved men with LUTS and benign prostatic hyperplasia (BPH). ${ }^{12}$ The goal of this clinical trial was to confirm that silodosin is superior to the placebo and not inferior to tamsulosin at a daily dose of $0.2 \mathrm{mg}$. The inclusion criteria were men aged $\geq 50$ years with an International Prostate Symptom Score (IPSS) of $\geq 8$, a quality-of-life (QoL) score of $\geq 3$, a maximum urinary flow rate $\left(\mathrm{Q}_{\max }\right)$ of $<15 \mathrm{~mL} / \mathrm{s}$, a prostate volume of $\geq 20 \mathrm{~mL}$, and a postvoid residual urine volume of $<100 \mathrm{~mL}$. Patients with a history of complications that would affect voiding were excluded from this study. Patients were randomized into three groups, with each group receiving silodosin at a dose of $4 \mathrm{mg}$ twice daily, tamsulosin at a dose of $0.2 \mathrm{mg}$ once daily, or the placebo for 12 weeks. The change in the IPSS from baseline was the primary endpoint. Secondary endpoints were the change in $\mathrm{Q}_{\max }$, urodynamic profiles, and the evaluation of subjective symptoms (eg, the IPSS storage and voiding scores, and the QoL score). Patient safety was assessed by a

Table I Summary of studies for uroselectivity of $\alpha$ I-adrenergic receptor antagonists

\begin{tabular}{|c|c|c|c|c|c|}
\hline & \multicolumn{2}{|c|}{$\begin{array}{l}\text { In vitro receptor } \\
\text { binding affinity }\end{array}$} & \multicolumn{2}{|c|}{ In vitro tissue selectivity } & \multirow{2}{*}{$\begin{array}{l}\text { In vivo ability to } \\
\text { suppress } P E \text { effect } \\
E_{15} / I_{50}\end{array}$} \\
\hline & $\alpha_{1} A / \alpha_{1} B$ & $\alpha_{1} D / \alpha_{1} B$ & Prostate/spleen & Prostate/aorta & \\
\hline Silodosin & 162 & 2.95 & 282 & 52 & 11.7 \\
\hline Tamsulosin $\mathrm{HCl}$ & 9.55 & 3.80 & 19 & 1.3 & 2.24 \\
\hline Naftopidil & 0.372 & 1.78 & 2.5 & 0.2 & 0.133 \\
\hline Prazosin $\mathrm{HCl}$ & 0.204 & 0.316 & 0.04 & 0.05 & 0.196 \\
\hline
\end{tabular}

Abbreviations: $\mathrm{HCl}$, hydrochloride; $\mathrm{PE}$, phenylephrine; $\mathrm{ID}_{50}$, defined as the dose at which intraurethral pressure is suppressed by $50 \%$; $E D_{15}$, defined as the dose at which the mean blood pressure is decreased by $15 \%$. 
Table 2 Characteristics of Phase III studies

\begin{tabular}{|c|c|c|c|}
\hline & $\begin{array}{l}\text { Kawabe et al } \\
\text { (Japanese study) }\end{array}$ & $\begin{array}{l}\text { Chapple et al }{ }^{14} \\
\text { (European study) }\end{array}$ & $\begin{array}{l}\text { Marks et al }{ }^{13} \\
\text { (American study) }\end{array}$ \\
\hline Total number of patients randomized & 457 & 955 & 923 \\
\hline \multirow[t]{3}{*}{ Intervention } & Silodosin 4 mg BID & Silodosin 8 mg daily & Silodosin 8 mg daily \\
\hline & Tamsulosin $0.2 \mathrm{mg}$ & Tamsulosin $0.4 \mathrm{mg}$ & Placebo \\
\hline & Placebo & Placebo & \\
\hline \multirow[t]{2}{*}{ Primary endpoint } & Change in IPSS & Change in IPSS from baseline & Change in IPSS from \\
\hline & from baseline & & baseline \\
\hline Study duration & 12 weeks & 12 weeks & 12 weeks \\
\hline \multicolumn{4}{|l|}{ Demographics } \\
\hline \multicolumn{4}{|l|}{ Mean age, years } \\
\hline Silodosin & 65.4 & 65.8 & 64.6 \\
\hline Tamsulosin & 65.6 & 65.9 & NA \\
\hline Placebo & 65.0 & 66.0 & 64.7 \\
\hline \multicolumn{4}{|l|}{ Total baseline IPSS, mean \pm SD } \\
\hline Silodosin & $17.1 \pm 5.7$ & $19.1 \pm 4.23$ & $21.3 \pm 5.1$ \\
\hline Tamsulosin & $17.0 \pm 5.7$ & $18.9 \pm 4.37$ & NA \\
\hline Placebo & $|7.1 \pm 6|$. & $19.3 \pm 4.33$ & $21.3 \pm 4.9$ \\
\hline
\end{tabular}

Abbreviations: IPSS, International Prostate Symptom Score; BID, twice a day; NA, not applicable; SD, standard deviation.

history of adverse events, physical examinations, vital signs, and laboratory tests.

Four hundred and fifty-seven patients received silodosin (176), tamsulosin (192), or the placebo (89). There were no significant differences in baseline characteristics, except for the QoL score, in the three groups. The mean standard deviation (SD) change in the total IPSS from

Table 3 Summary of prospective randomized, placebo-controlled studies on silodosin

- A significant decrease in the IPSS compared to the placebo in the silodosin group at I week (J)

- A significant decrease in the IPSS compared to tamsulosin at 2 weeks (J)

- In patients with severe symptoms (IPSS $\geq 20$ ), silodosin shows a significant improvement of IPSS compared to the placebo $(-12.4$ versus -8.7$)(J)$

- Silodosin is superior to the placebo in IPSS storage and voiding subscore analyses $(E)$

- Silodosin significantly reduces nocturia compared to the placebo $(-0.9$ versus $-0.7, P=0.013)$ ( $E$ )

- Differences of improvements in the total IPSS and subscores between silodosin and placebo increased by week 12 (A)

- Mean change of the $Q_{\text {max }}$ at 2-6 hours after the initial dose is greater with silodosin than with the placebo ( 2.8 versus I.5, $P<0.000 \mathrm{I}$ ) (A)

- Long-term treatment of up to 52 weeks with silodosin shows sustained improvement of BPH symptoms (E, A)

- Silodosin significantly increases the chances of a successful trial without catheter (TWOC) after acute urinary retention ${ }^{16}$

- QD administration of silodosin (8 $\mathrm{mg}$ once daily) is not inferior to BID administration (4 mg twice daily) in efficacy and safety studies ${ }^{6}$

Notes: J, Japanese Phase III trial; E, European Phase III trial; A, American Phase III trial; Kumar et al; ${ }^{16}$ Choo et al. ${ }^{6}$

Abbreviations: BPH, benign prostatic hyperplasia; IPSS, International Prostate Symptom Score. baseline was $-8.3(6.4),-6.8(5.7)$, and -5.3 (6.7) for silodosin, tamsulosin, and the placebo, respectively. At 1 , and 2 weeks post-treatment, there was a more significant decrease in the IPSS in the silodosin group compared to placebo and tamsulosin groups, respectively. The mean ( $95 \%$ confidence interval $[\mathrm{CI}]$ ) intergroup differences in the total IPSS between silodosin and placebo groups, and between silodosin and tamsulosin groups were -3.0 (-4.6, -1.3), and $-1.4(-2.7,-0.2)$, respectively. The mean (SD) change in the QoL score from baseline was -1.7 (1.4), -1.4 (1.3), and -1.1 (1.2) in silodosin, tamsulosin, and placebo groups, respectively. The improvement of QoL score $(P=0.002)$ was highest in the silodosin group. Silodosin treatment also improved storage and voiding symptoms compared to the placebo. In addition, silodosin showed significant improvements in the total IPSS when compared to the placebo in patients with moderate (IPSS 8-19) to severe (IPSS $\geq 20$ ) symptoms. In the original study, ${ }^{12}$ the silodosin group did not show a significant improvement in the $\mathrm{Q}_{\max }$ compared to the placebo. The authors have speculated that immense changes in voiding volume before and after treatment in some men may have affected the results. So, a post hoc investigation was conducted in the overall subgroup of patients whose difference of voided volume between pre- and post-treatment was less than $50 \%$. The mean (SD) change in $\mathrm{Q}_{\max }$ from baseline was $1.70(3.31), 2.60(3.98)$, and $0.26(2.21) \mathrm{mL} / \mathrm{s}$ in the silodosin, tamsulosin, and placebo groups, respectively, and silodosin was significantly $(P=0.005)$ better than the placebo in improving $\mathrm{Q}_{\max }$. 
The incidence of drug-related adverse events was $69.7 \%$, $47.4 \%$, and $36.4 \% \%$ for silodosin, tamsulosin, and the placebo, respectively, indicating that the silodosin group had the highest frequency $(P<0.001)$ of adverse events. Eighteen (10.2\%), 11 $(5.7 \%)$, and four $(4.5 \%)$ patients in silodosin, tamsulosin, and placebo groups, respectively, discontinued treatment. While these adverse events were resolved after terminating treatment, the most common adverse event after silodosin treatment was abnormal ejaculation. Five men (2.9\%) discontinued treatment due to this problem. There were no clinically significant differences in the systolic/diastolic blood pressure or heart rate between silodosin and tamsulosin groups.

A larger, international, randomized, double-blind, placebo-, and active-controlled clinical trial was conducted in 72 European centers. ${ }^{14}$ Patients in this study received silodosin at a dose of $8 \mathrm{mg}$, tamsulosin at a dose of $0.4 \mathrm{mg}$, or the placebo once daily for 12 weeks. The inclusion criteria were men aged $\geq 50$ years with an IPSS of $\geq 13$, and a $\mathrm{Q}_{\max }$ of $>4 \mathrm{~mL} / \mathrm{s}$ and $\leq 15 \mathrm{~mL} / \mathrm{s}$. Approximately $60 \%$ of patients were elderly, and approximately $57 \%$ of patients were on concomitant antihypertensive medication. After a 2:2:1 randomization of 955 patients into silodosin ( $8 \mathrm{mg}, \mathrm{n}=381)$, tamsulosin (0.4 mg, $n=384)$, or placebo $(n=190)$ groups, the authors assessed the change from baseline in the total IPSS (primary endpoint), storage and voiding subscores, the QoL score due to urinary symptoms, and the $\mathrm{Q}_{\max }$. Responders were defined by a $\geq 25 \%$ decrease in the IPSS and $\mathrm{a} \geq 30 \%$ increase in the $\mathrm{Q}_{\max }$ from baseline. In this study, the change in the total IPSS after silodosin $(-2.3$ $[95 \% \mathrm{CI},-3.2,-1.4])$ and tamsulosin $(-2.0[95 \% \mathrm{CI},-2.9,-1.1])$ was significantly greater than that of the placebo $(P<0.001)$. The responder rate according to the total IPSS was significantly higher $(P<0.001)$ in silodosin $(66.8 \%)$ and tamsulosin $(65.4 \%)$ groups than in the placebo $(50.8 \%)$. Active treatments were also superior to the placebo in the IPSS, storage, and voiding subscore analyses, and the QoL score due to urinary symptoms. Although there was no statistically significant difference in storage and voiding subscores between silodosin and tamsulosin groups, only silodosin significantly reduced nocturia compared to the placebo (the changes from baseline were $-0.9,-0.8$, and -0.7 for silodosin, tamsulosin, and the placebo, respectively; $P=0.013$ for silodosin versus (vs) placebo, $P=0.095$ for tamsulosin vs placebo, and $P=0.314$ for silodosin vs tamsulosin). Active treatments did not show superiority in the $Q_{\max }$ because all groups showed a similar degree of improvement. The adjusted mean change from baseline to the endpoint was $3.77 \mathrm{~mL} / \mathrm{s}$ for silodosin, $3.53 \mathrm{~mL} / \mathrm{s}$ for tamsulosin, and $2.93 \mathrm{~mL} / \mathrm{s}$ for the placebo. The percentage of $\mathrm{Q}_{\max }$ responders was $46.6 \%, 46.5 \%$, and $40.5 \%$ for silodosin, tamsulosin, and the placebo, respectively.
Active treatments were well-tolerated. The most frequent adverse event with silodosin was the reduction or absence of ejaculation during orgasm (14\%), which is characteristic of selective $\alpha 1 \mathrm{~A}$-adrenoceptor antagonists. Its incidence was higher than that observed with tamsulosin (2.1\%) and the placebo (1.1\%). Reported adverse effects relating to ejaculation were orgasms with no semen, a reduced semen quantity during orgasm, and retrograde ejaculation. There was also a higher incidence of headaches in the tamsulosin group (5.5\%, 21 out of 384$)$ compared with the silodosin group $(2.9 \%, 11$ out of 381 ), but this incidence was not significantly different compared to the placebo $(4.7 \%, 9$ out of 190$)$. There was no statistically significant difference among groups in the percentage of patients who discontinued the study due to adverse events ( $2.1 \%$ for silodosin, eight subjects, $1.0 \%$ for tamsulosin, four subjects, and $1.6 \%$ for the placebo, three subjects). The most common cause of discontinuation was the failure to ejaculate (five subjects in the silodosin group, one in the tamsulosin group), but this adverse effect was reversed after study termination. In terms of adverse cardiovascular events, there were no meaningful changes in laboratory parameters, vital signs, or electrocardiography (ECGs) in all groups. Whereas a minor, but statistically significant difference in incidence of cardiovascular event was noted in the tamsulosin group compared with the placebo, silodosin did not affect blood pressure or heart rate. The change from baseline in the supine systolic blood pressure after silodosin or tamsulosin treatment was $-1.8(P=0.075)$, and $-2.2 \mathrm{mmHg}(P=0.022)$, respectively, which was significantly higher than the placebo $(-0.4 \mathrm{mmHg})$. The change from baseline in the supine diastolic blood pressure after silodosin treatment was not significantly different compared to the placebo $(-1.0$ and $-0.6 \mathrm{mmHg}$, respectively, $P=0.515)$, however, it was significantly higher after tamsulosin treatment $(-1.6 \mathrm{mmHg}, P=0.06)$. Moreover, there was no change from baseline in heart rate after treatment with silodosin, tamsulosin, or the placebo $(0.8,1.3,1.1$ beats per minute; $P>0.05)$.

Marks et al described results from two short-term Phase III studies performed in the United States on the safety and efficacy of silodosin. ${ }^{13}$ These two 12 -week studies were identically designed, parallel grouped, multicenter, randomized, doubleblind, and placebo-controlled. These studies included men aged $\geq 50$ years with an IPSS of $\geq 13, \mathrm{Q}_{\max }$ of $4-15 \mathrm{~mL} / \mathrm{sec}$, and a postvoid residual volume of $<250 \mathrm{~mL}$. After a 4 week placebo period, patients were randomized into either the silodosin or the placebo group. Patients with at least a 30\% decrease in IPSS or an increase in $\mathrm{Q}_{\max }$ of $3 \mathrm{~mL} / \mathrm{sec}$ or greater during the placebo period were excluded. The primary endpoint 
was a change in the IPSS from baseline at the last observation, the secondary endpoint was a change in the $\mathrm{Q}_{\max }$.

Out of 923 patients (mean age, 65 years) in this study, 466 patients received silodosin, and 457 patients received the placebo. After only 0.5 week (range, 3-4 days) of treatment, there were improvements in the total IPSS (difference $-1.9, P<0.0001)$, and storage $(-0.5, P<0.0002)$ and voiding $(-1.4, P<0.0001)$ subscores in patients receiving silodosin. The mean \pm SD change from baseline in the total IPSS was $-4.2 \pm 5.3$ for silodosin and $-2.3 \pm 4.4$ for the placebo. At 12 weeks, the differences of the improvement in the IPSS, and storage and voiding subscores became more significant (total IPSS $-6.4 \pm 6.63,-3.5 \pm 5.84$; storage subscore, $-2.3 \pm 2.93,-1.4 \pm 2.66$; voiding subscore $-4.0 \pm 4.31,-2.1 \pm 3.76$ in silodosin and placebo group, respectively; $(P<0.0001)$.

The mean change from baseline in the $\mathrm{Q}_{\max }(\mathrm{mL} / \mathrm{sec})$ at 2-6 hours after the initial dose was greater $(P<0.0001)$ with silodosin $(2.8 \pm 3.4)$ than with the placebo $(1.5 \pm 3.8)$. Differences remained significant $(P<0.001)$ through week 12 . During the course of the study, the improvement of the QoL score was greater in patients receiving silodosin than in those receiving the placebo. The most common adverse event was mild retrograde ejaculation $(28.1 \%$ for silodosin, $0.9 \%$ for the placebo), and only $2.8 \%$ of patients terminated treatment because of retrograde ejaculation. The percentage of patients needed treatment for orthostatic hypotension was $2.6 \%$ and $1.5 \%$ in silodosin and placebo group, respectively. Frequently noted adverse effects in patients treated with silodosin or the placebo are summarized in Table 4.

Ding et al summarized the safety and efficacy of the four randomized 12-week studies. ${ }^{15}$ In this report, the authors included 2,504 patients. Compared to the placebo, the authors showed a significant difference of improvement from baseline for the IPSS, the QoL score, and the $\mathrm{Q}_{\max }$ (mean difference [MD] of improvement $=-2.78$ in IPSS, $P<0.00001 ;-0.42$ in QoL, $P=0.004 ; 1.17$ in $\mathrm{Q}_{\max }, P<0.00001$, respectively) in

Table 4 Frequently noted adverse effects of silodosin compared to the placebo

\begin{tabular}{lll}
\hline & Silodosin & Placebo \\
\hline Retrograde ejaculation, \% range & I4.2-28.I & $0-1 . \mathrm{I}$ \\
Thirst, \% & 10.3 & 4.5 \\
Loose stool, \% & 9.1 & 5.6 \\
Dizziness, \% range & $3.2-5.1$ & 4.5 \\
Orthostatic hypotension, \% & 2.6 & 1.5 \\
Headache, \% range & $2.4-5.5$ & $0.9-4.7$ \\
Ejaculatory disorder leading to & $1.3-2.9$ & 0 \\
discontinuation of the study, \% range & & \\
\hline
\end{tabular}

patients treated with silodosin. Patients receiving silodosin also reported a higher patient satisfaction (higher QoL) with regard to storage and voiding. Most patients reported abnormal ejaculation as an adverse effect. Compared with tamsulosin at a dose of $0.2 \mathrm{mg}$, silodosin was ideal in relation to the IPSS and the QoL score (MD of improvement $=-1.14$ in IPSS, $P=0.02 ;-0.26$ in QOL, $P=0.02$ ), but inferior with regard to the $\mathrm{Q}_{\max }\left(-0.85 \mathrm{Q}_{\max }, P=0.01\right)$. By contrast, there was no significant difference in the incidence of abnormal ejaculation or dizziness between the silodosin and tamsulosin groups.

\section{Other Asian studies}

Kumar et al investigated the safety and efficacy of silodosin in the management of acute urinary retention in a prospective randomized placebo-controlled study. ${ }^{16}$ In this study, 60 men aged $>50$ years with acute urinary retention were randomized into either the silodosin group ( $8 \mathrm{mg}$ once daily) or the placebo group for 3 days, followed by trial without catheter (TWOC). In this study, a failed TWOC was defined as the presence of urinary retention or a postvoid residual urine volume of $>150 \mathrm{~mL}$, and all patients with a successful TWOC on day 3 were started on silodosin. The TWOC success rate in the silodosin group was $76.7 \%$; in the placebo group, it was $36.7 \%(P=0.002)$. Multivariate analysis showed the odds of failing TWOC to be lower in patients receiving silodosin (0.13) compared to those receiving the placebo $(P=0.008)$. Among the clinical parameters, a retention volume of $>800 \mathrm{~mL}(P=0.038)$ and an IPSS score of $>25(P=0.042)$ had significantly greater odds of failure. No adverse effects were reported in this study.

$\mathrm{Yu}$ et al reported results from a non-inferiority study of silodosin and tamsulosin performed in nine medical centers in Taiwan. ${ }^{17}$ Two hundred and nine men at 40 years of age with an IPSS of $\geq 13$, a QoL score of $\geq 3$, a prostate volume of $\geq 20 \mathrm{~mL}$, and a $\mathrm{Q}_{\max }$ of $<15 \mathrm{~mL} / \mathrm{s}$ with a voided volume of $\geq 100 \mathrm{~mL}$ were enrolled. The authors assessed the change from baseline in the IPSS. The non-inferiority margin of the IPSS change was set at 1.0. The change in the $\mathrm{Q}_{\max }$ and QoL score was also assessed. The mean difference (silodosin minus tamsulosin) of changes in the IPSS from baseline was -0.60 (95\% CI: -2.15 to 0.95$)$, which indicates that silodosin was non-inferior to tamsulosin. The mean changes in the $\mathrm{Q}_{\max }$ and QoL score from baseline were comparable between both groups $(P<0.05)$. Patients receiving silodosin had a significantly higher incidence of abnormal ejaculation $(9.7 \%$ for silodosin vs $1.0 \%$ for tamsulosin, $P<0.009$ ), but only $1.9 \%$ of patients discontinued treatment. 
Tamsulosin significantly reduced the mean systolic blood pressure $(-4.2 \mathrm{mmHg}$, within-group $P<0.004)$ compared to the negligible change of silodosin $(-0.1 \mathrm{mmHg}$, withingroup $P=0.96$ ).

Choo et al describe a 12 -week, double-blind, randomized, parallel, and multicenter study. ${ }^{6}$ They compared the safety and efficacy of silodosin QD (8 mg once daily) with silodosin BID (4 mg twice daily) in Korean men ( $n=424)$, and assessed the change from baseline in the total IPSS at 12 weeks. Adverse drug reactions and vital signs, and laboratory test results were also recorded. The change in the total IPSS in the QD group was not inferior to that of the BID group $(-6.70$ and -6.94 , respectively; 95\% CI, -0.88 to 1.36 ). Moreover, there were no significant differences in the percentages of IPSS of patients with $a \geq 25 \%$ (63.41\% and $67.82 \%$, respectively; $P=0.349$ ) or $\geq 4$-point improvement in the total IPSS $(65.85 \%$ and $69.31 \%$, respectively; $P=0.457$ ) and a $\geq 30 \%$ improvement in the $\mathrm{Q}_{\max }(47.32 \%$ and $40.59 \%$, respectively; $P=0.172)$. There were also no changes in the following parameters: the IPSS voiding subscore $(-4.42 \pm 4.93$ and $-4.65 \pm 4.77 ; P=0.641)$, the IPSS storage subscore $(-2.05 \pm 3.07$ and $-2.52 \pm 2.97$; $P=0.117)$, the QoL score $(-1.19 \pm 1.49$ and $-1.40 \pm 1.42$; $P=0.136)$, the $\mathrm{Q}_{\max }(3.55 \pm 5.93$ and $3.74 \pm 6.79 \mathrm{~mL} / \mathrm{s}$; $P=0.768)$, the International Continence Society male questionnaire score, the Patient Goal Achievement Score, and the Treatment Satisfaction Questionnaire. The two groups had similar frequencies of adverse drug reactions. The authors concluded that there was no difference between administration routes.

\section{Long-term treatment}

In Europe and the United States, 12-week, double-blind, Phase III studies and 40-week, non-comparative extension trials with open-label silodosin $8 \mathrm{mg}$ /day were conducted. ${ }^{18,19}$ Six hundred and sixty-one men enrolled in extension trials conducted in the United States, ${ }^{19}$ and 509 men entered similar studies in Europe. ${ }^{18}$ After assessing the long-term tolerability and efficacy (up to 52 weeks) of silodosin, patients reported relief from BPH symptoms. Moreover, patients previously treated with silodosin maintained the total IPSS during the open-label period. During the 40-week extension trials, there was a moderate decrease in the total IPSS ( -1.0 from baseline in the American trial, $P<0.01$ and -0.82 from baseline in the European trial, $P$-value not stated). Patients who had previously received the placebo achieved a decrease of $>2$ points in the total IPSS after changing to open-label silodosin treatment $(-3.0$ in the
American trial, $P<0.001$ and -2.7 in the European trial, $P$-value not stated).

\section{Abnormal ejaculation and symptom improvement}

As summarized in Table 4, silodosin is associated with several side effects. Among these, abnormal ejaculation was the most commonly reported symptom. Silodosin-mediated abnormal ejaculation may involve two mechanisms. Firstly, the inhibition of $\alpha 1 \mathrm{~A}$-adrenergic receptor expression in the bladder neck, prostate, and urethra caused by silodosin may relax these organs, resulting in retrograde ejaculation. ${ }^{20}$ Secondly, silodosin inhibits semen emission, which can result in abnormal ejaculation. ${ }^{21,22}$ Although discontinuation of studies due to abnormal ejaculation was relatively low (7.1\% in an observational study), ${ }^{23}$ some patients experienced frustration with this symptom. Therefore, patients should be educated on the adverse effects of silodosin treatment. On the other hand, Phase III studies suggested that the existence of ejaculatory disorder was correlated with the magnitude of symptom improvement in the patients treated with silodosin. ${ }^{24,25}$ Nevertheless, abnormal ejaculation caused by silodosin improves LUTS.

\section{Conclusion}

$\alpha 1$-adrenergic receptor antagonists are commonly used to treat male LUTS and BPH. Silodosin is a novel $\alpha 1$-adrenergic receptor antagonist whose affinity for the $\alpha 1 \mathrm{~A}$-adrenergic receptor is greater than that for the $\alpha 1 \mathrm{~B}$-adrenergic receptor. Silodosin seemed not to affect the blood pressure and heart rate compared to the placebo. Silodosin at a daily dose of $8 \mathrm{mg}$ was effective, improving the IPSS and $\mathrm{Q}_{\max }$. Silodosin was also safe and effective in the long-term treatment of nocturia. Retrograde or abnormal ejaculation was the most commonly reported side effect in the patients treated with silodosin, however, discontinuation of Phase III studies due to abnormal ejaculation was relatively low. Evidence showing solid efficacy and cardiovascular safety profiles of silodosin will provide a good solution for the treatment of LUTS associated with BPH in an increasingly aging society.

\section{Disclosure}

The authors report no conflicts of interest in this work.

\section{References}

1. Yoshida M, Kudoh J, Homma Y, Kawabe K. New clinical evidence of silodosin, an $\alpha(1 \mathrm{~A})$ selective adrenoceptor antagonist, in the treatment for lower urinary tract symptoms. Int J Urol. 2012;19(4): 306-316. 
2. Shibata K, Foglar R, Horie K, et al. KMD-3213, a novel, potent, alpha 1a-adrenoceptor-selective antagonist: characterization using recombinant human alpha 1 -adrenoceptors and native tissues. Mol Pharmacol. 1995;48(2):250-258.

3. Moriyama N, Akiyama K, Murata S, et al. KMD-3213, a novel alpha1A-adrenoceptor antagonist, potently inhibits the functional alpha1-adrenoceptor in human prostate. Eur J Pharmacol. 1997;331(1): 39-42.

4. Akiyama K, Tatemichi S, Katayama S, et al. Relationship between prostatic alpha(1)-adrenoceptor binding and reduction in intraurethral pressure following continuous infusion of KMD-3213 in rats. Pharmacology. 2002;64(3):140-147.

5. Tatemichi S, TomiyamaY, Maruyama I, et al. Uroselectivity in male dogs of silodosin (KMD-3213), a novel drug for the obstructive component of benign prostatic hyperplasia. Neurourol Urodyn. 2006;25(7): 792-799; discussion 800-801.

6. Choo MS, Song M, Kim JH, et al. Safety and efficacy of 8-mg oncedaily vs 4-mg twice-daily silodosin in patients with lower urinary tract symptoms suggestive of benign prostatic hyperplasia (SILVER Study): a 12-week, double-blind, randomized, parallel, multicenter study. Urology. 2014;83(4):875-881

7. Tatemichi S, Kobayashi K, Maezawa A, Kobayashi M, Yamazaki Y, Shibata N. [Alpha1-adrenoceptor subtype selectivity and organ specificity of silodosin(KMD-3213)]. Yakugaku zasshi. 2006;126 Spec no:209-216. Japanese.

8. Ishiguro M, Futabayashi Y, Ohnuki T, Ahmed M, Muramatsu I, Nagatomo T. Identification of binding sites of prazosin, tamsulosin and KMD-3213 with alpha(1)-adrenergic receptor subtypes by molecular modeling. Life Sci. 2002;71(21):2531-2541.

9. Tatemichi S, Kobayashi K, Maruyama I, Kobayashi M, Yamazaki Y, Shibata N. [Effects of silodosin (KMD-3213) on phenylephrine-induced increase in intraurethral pressure and blood pressure in rats - study of the selectivity for lower urinary tract]. Yakugaku zasshi. 2006;126 Spec no:217-223. Japanese.

10. Yoshida M, Homma Y, Kawabe K. Silodosin, a novel selective alpha 1A-adrenoceptor selective antagonist for the treatment of benign prostatic hyperplasia. Expert Opin Investig Drugs. 2007;16(12): 1955-1965.

11. MacDiarmid SA, Hill LA, Volinn W, Hoel G. Lack of pharmacodynamic interaction of silodosin, a highly selective alpha1a-adrenoceptor antagonist, with the phosphodiesterase-5 inhibitors sildenafil and tadalafil in healthy men. Urology. 2010;75(3):520-525.

12. Kawabe K, Yoshida M, Homma Y, Silodosin Clinical Study Group. Silodosin, a new alpha1A-adrenoceptor-selective antagonist for treating benign prostatic hyperplasia: results of a phase III randomized, placebo-controlled, double-blind study in Japanese men. BJU Int. 2006;98(5):1019-1024.
13. Marks LS, Gittelman MC, Hill LA, Volinn W, Hoel G. Rapid efficacy of the highly selective alpha1A-adrenoceptor antagonist silodosin in men with signs and symptoms of benign prostatic hyperplasia: pooled results of 2 phase 3 studies. J Urol. 2009;181(6):2634-2640.

14. Chapple CR, Montorsi F, Tammela TL, Wirth M, Koldewijn E, Fernandez Fernandez E, European Silodosin in Study Group. Silodosin therapy for lower urinary tract symptoms in men with suspected benign prostatic hyperplasia: results of an international, randomized, doubleblind, placebo- and active-controlled clinical trial performed in Europe. Eur Urol. 2011;59(3):342-352.

15. Ding H, Du W, Hou ZZ, Wang HZ, Wang ZP. Silodosin is effective for treatment of LUTS in men with BPH: a systematic review. Asian J Androl. 2013;15(1):121-128.

16. Kumar S, Tiwari DP, Ganesamoni R, Singh SK. Prospective randomized placebo-controlled study to assess the safety and efficacy of silodosin in the management of acute urinary retention. Urology. 2013;82(1): $171-175$.

17. Yu HJ, Lin AT, Yang SS, et al. Non-inferiority of silodosin to tamsulosin in treating patients with lower urinary tract symptoms (LUTS) associated with benign prostatic hyperplasia (BPH). BJU Int. 2011;108(11): 1843-1848.

18. Curran MP. Silodosin: treatment of the signs and symptoms of benign prostatic hyperplasia. Drugs. 2011;71(7):897-907.

19. Marks LS, Gittelman MC, Hill LA, Volinn W, Hoel G. Silodosin in the treatment of the signs and symptoms of benign prostatic hyperplasia: a 9-month, open-label extension study. Urology. 2009;74(6): $1318-1322$.

20. Michel MC. Alpha1-adrenoceptors and ejaculatory function. $\mathrm{Br} J$ Pharmacol. 2007;152(3):289-290.

21. Kobayashi K, Masumori N, Hisasue S, et al. Inhibition of Seminal emission is the main cause of anejaculation induced by a new highly selective alpha1 A-blocker in normal volunteers. $J$ Sex Med. 2008;5(9): 2185-2190.

22. Hisasue S, Furuya R, Itoh N, Kobayashi K, Furuya S, Tsukamoto T. Ejaculatory disorder caused by alpha-1 adrenoceptor antagonists is not retrograde ejaculation but a loss of seminal emission. Int J Urol. 2006;13(10):1311-1316.

23. Descazeaud A, Davin JL, De La Taille A, et al. [Evolution of lower urinary tract symptoms under silodosin: Factors influencing patients' satisfaction]. Progres Urol. 2014;24(3):196-202. French.

24. Homma Y, Kawabe K, Takeda M, Yoshida M. Ejaculation disorder is associated with increased efficacy of silodosin for benign prostatic hyperplasia. Urology. 2010;76(6):1446-1450.

25. Roehrborn CG, Kaplan SA, Lepor H, Volinn W. Symptomatic and urodynamic responses in patients with reduced or no seminal emission during silodosin treatment for LUTS and BPH. Prostate Cancer Prostatic Dis. 2011;14(2):143-148.
Research and Reports in Urology

\section{Publish your work in this journal}

Research and Reports in Urology is an international, peer-reviewed, open access journal publishing original research, reports, editorials, reviews and commentaries on all aspects of adult and pediatric urology in the clinic and laboratory including the following topics: Pathology, pathophysiology of urological disease; Investigation and treatment of

\section{Dovepress}

urological disease; Pharmacology of drugs used for the treatment of urological disease. The manuscript management system is completely online and includes a very quick and fair peer-review system, which is all easy to use. Visit http://www.dovepress.com/testimonials.php to read real quotes from published authors. 